\title{
Genetic variants affecting the neural processing of human facial expressions: evidence using a genome-wide functional imaging approach
}

\author{
AA Brown ${ }^{1,2,11}$, J Jensen ${ }^{1,3,4,11}$, YS Nikolova ${ }^{5}$, S Djurovic ${ }^{1,6}$, I Agartz $^{1,7}$, A Server ${ }^{8}$, RE Ferrell ${ }^{9}$, SB Manuck ${ }^{9}$, M Mattingsdal ${ }^{1,10}$, \\ I Melle ${ }^{1,3}$, AR Hariri $^{5}$, A Frigessi $^{2}$ and OA Andreassen ${ }^{1,3}$
}

Human faces present crucial visual information for social interaction. Specialized brain regions are involved in the perception of faces, with the fusiform face area (FFA) a key neuronal substrate. Face processing is genetically controlled, but by which specific genes is unknown. A genome-wide approach identified common single nucleotide polymorphisms (SNPs) associated with areas of increased brain activity in response to affective facial expressions, measured with functional magnetic resonance imaging. SNPs in $\mathbf{2 0}$ genetic regions were linked with neural responses to negative facial expressions in a Norwegian sample $(n=246)$, which included patients with mental illness. Three genetic regions were linked with FFA activation in a further discovery experiment using positive facial expressions and involving many of the same individuals $(n=284)$. Two of these three regions showed significant association with right FFA activation to negative facial expressions in an independent North American replication sample of healthy Caucasians $(n=85,3 q 26.31, P=0.004 ; 20 \mathrm{p} 12.3, P=0.045)$. The activation patterns were particularly striking for the SNP in 3q26.31, which lies in a gene TMEM212; only the FFA was activated. The specialized function of this brain region suggests that TMEM212 could contribute to the innate architecture of face processing.

Translational Psychiatry (2012) 2, e143; doi:10.1038/tp.2012.67; published online 24 July 2012

\section{Introduction}

Face processing is a crucial cognitive ability. Rapid recognition of faces identifies individuals and informs us about basic properties such as age, gender and race, as well as complex processes such as direction of attention, mood and intent. Thus, the ability to extract such information from even a momentary viewing of a face is critically important for normal social interactions.

Several lines of evidence indicate that face processing is unique and qualitatively different from processing of other visual stimuli. ${ }^{1,2}$ Studies of neurological disorders suggest a dedicated circuitry exists in the human brain for face processing. Converging evidence now suggests that a network of multiple brain regions, in particular the right fusiform gyrus, supports face perception. ${ }^{3-6}$

Similar specialized processing of faces has been reported in macaques, ${ }^{7,8}$ and it has been suggested that specialized pathways for face processing were critical for the survival of our primate ancestors. ${ }^{9}$ This implies that face processing is under some genetic control, and findings in infants show that face recognition is an innate human capability. ${ }^{10,11}$ In fact, using behavioral tasks several twin studies have detected a high degree of heritability in face processing ${ }^{12,13}$ (one study found that correlation of one particular score of face recognition for monozygotic twins $(0.70)$ was more than double that of dizygotic twins correlation $(0.29)^{13}$ ). However, the specific genes involved in face processing are unknown, as is the effects of such genes on the neural networks supporting this specialized behavior.

The aim of the current study was to identify common genetic variants associated with neural activation during face processing. Using genome-wide genotype microarray technology together with functional magnetic resonance imaging (fMRI) in an ethnically homogenous sample of healthy controls and psychiatric patients from Norway, ${ }^{14}$ and an independent replication sample of Caucasians from North America, ${ }^{15}$ we discovered two genomic regions associated with neural activation during face processing.

\section{Materials and methods}

The Norwegian TOP sample consisted of 246 individuals after quality control on the genotype and imaging data. The fMRI protocol consisted of a widely utilized emotional faces paradigm, ${ }^{15-17}$ involving the perceptual processing (matching) of

\footnotetext{
${ }^{1}$ Division of Mental Health and Addiction, Institute of Clinical Medicine, University of Oslo, Oslo, Norway; ${ }^{2}$ Department of Biostatistics, Institute of Basic Medical Sciences, University of Oslo, Oslo, Norway; ${ }^{3}$ Division of Mental Health and Addiction, Oslo University Hospital, Oslo, Norway; ${ }^{4}$ Department of Psychiatry and Psychotherapy, Charité Universitätsmedizin, Berlin, Germany; ${ }^{5}$ Department of Psychology \& Neuroscience, Institute for Genome Sciences \& Policy, Duke University, Durham, NC, USA; ${ }^{6}$ Department of Medical Genetics, Oslo University Hospital, Oslo, Norway; ${ }^{7}$ Department of Research and Development, Diakonhjemmet Hospital, Oslo, Norway; ${ }^{8}$ Department of Neuroradiology, Oslo University Hospital, Oslo, Norway; ${ }^{9}$ Departments of Psychology and Human Genetics, University of Pittsburgh, Pittsburgh, PA, USA and ${ }^{10}$ Research Unit, Sorlandet Hospital HF, Kristiansand, Norway

Correspondence: Dr AA Brown, Department of Biostatistics, Institute of Basic Medical Sciences, University of Oslo, PO Box 1122, Blindern, N-0317 Oslo, Norway. E-mail: andrew.brown@medisin.uio.no

${ }^{11}$ These authors contributed equally to this work.

Keywords: face processing; fMRI; genome-wide association

Received 28 September 2011; revised 8 May 2012; accepted 31 May 2012
} 
emotional facial expressions (experimental condition) or geometric shapes (control condition). Subjects completed two sessions, one with all negative (angry or afraid) and another all positive (happy) expressions, in a counterbalanced order. We will refer to these experiments as the negative and positive faces experiments. No one was excluded based on performance as it was a very easy task and subjects practiced it to demonstrate understanding before entering the scanner. In the negative faces experiment, the age was $34 \pm 10$ (mean \pm s.d.) years and there were 130 men and 116 women. There were 138 participants with mental disorder, whereas 108 were healthy controls. For the positive faces paradigm, we included 284 individuals, age $34 \pm 9$ years; 157 male, 127 female, 160 with mental disorder and 124 healthy controls. Single nucleotide polymorphism (SNP) genotyping was performed using the Affymetrix Gene Chip Genome Wide SNP 6.0 array (Affymetrix Inc., Santa Clara, CA, USA, http://www.affymetrix.com). Individuals with discrepancies between reported and genotype-inferred gender and those whose ancestry differed from the majority of the sample were removed. Mitochondrial and X-chromosome SNPs were also removed, and we excluded individuals and SNPs with $>3 \%$ missing data, and SNPs with a minor allele frequency of $<0.05$.

The fMRI data from both tasks were processed using the SPM2 software ${ }^{18}$ to produce contrast maps. For both negative and positive faces experiments, we focused on the 20,000 voxels with most evidence of differential activation to reduce multiple testing. A further prescreening stage rejected voxels in clusters of $<50$. In the negative faces experiment, every voxel was tested for association with every SNP using an additive model of genetic effect. For each voxel, the $P$-value for the most significant SNP was adjusted for multiple testing across the SNPs using the Šidák correction. ${ }^{19}$ Multiple testing across the voxels was accounted for by controlling the false discovery rate (FDR) under the assumption of positive dependencies using a common adjustment method. ${ }^{20}$ We validated our associations in the positive faces experiment by testing each voxel for association with 35 SNPs suggested by the negative faces experiment; multiple testing across voxels was controlled for each SNP separately. ${ }^{20}$ In both analyses, voxels were only included in the results if they lay within clusters of $>10$.

The North American replication sample consisted of 85 healthy middle-aged Caucasian subjects, 41 males, 44 females; age $45 \pm 7$ years). All fMRI data preprocessing and single-subject analyses were completed using SPM2. ${ }^{18}$ These participants were genotyped using the Illumina 610Quad BeadChip (Illumina Inc., San Diego, CA, USA). Perfect proxies were available on this array in two of the genetic regions under study, while the third region had no proxy and so was separately genotyped using fluorescence polarization methods. Single-subject contrast images were entered into separate regression analyses with the rs 12485367 , rs6038686 and rs6081495 genotypes, including gender and age as nuisance covariates; these analyzes were conducted in SPM8 ${ }^{18}$ (http://www.fil.ion.ucl.ac.uk/spm).

More details, including a full description of the subjects, and the processing and analysis of the data can be found in Supplementary material available online.

\section{Results}

Faces task in Norwegian sample. We carried out genomewide analyses using the Affymetrix 6.0 Array on the subjects who had successfully completed the fMRI protocol. Clusters of voxels exhibiting significant differential activation in the direct comparisons of the experimental and control conditions were used. The 20,000 most significant voxels were identified and any clusters of $<50$ voxels were discarded, in order to choose groups of voxels able to contain diffuse associations. After this procedure, 19,742 voxels remained for analysis from the negative faces scan, and 19,864 from the positive faces scan. For both the negative and positive faces, voxels exhibiting a significant main effects of task (that is, experimental $>$ control condition) were principally located in ventral visual cortices as well as frontal and subcortical regions. There is highly significant evidence of differential activation for these voxels (Bonferroni-corrected family-wise error (FWE) rate for the voxels in the negative faces is $\left.1.2 \times 10^{-8}\right)$. The exact location of the voxels selected for both scans is provided in Supplementary Figure S1 online.

Genetic variants suggested by negative faces experiment. Initially, fMRI data from the scan with negative facial expressions were analyzed as these stimuli have been more commonly used than positive expressions in the larger neuroimaging literature. ${ }^{21}$ Each of the voxels as chosen above was tested for an association with each SNP (a total of 549,640 SNPs, meaning approximately $10^{10}$ tests) using an additive model of genetic effect. The $P$-values were adjusted to account for multiple testing: first using the Šidák correction ${ }^{19}$ to produce $P$-values, adjusted for multiple testing across the SNPS. Then the Benjamini Hochberg procedure ${ }^{20}$ was applied to these adjusted $P$-values to control the FDR (the procedure controls the expected proportion of false positive results), accounting for multiple testing across both SNPs and voxels. With the exception of the preselection of task-related voxels described above, this is a similar strategy to one previously presented applied in a genome-wide association study of structural imaging phenotypes. ${ }^{22}$ In that paper, identical procedures for controlling for multiple testing across SNPs and phenotypes (vertices as opposed to voxels) were followed, with the exception that we applied a more conservative procedure to control for multiple testing across SNPs (ignoring linkage disequilibrium structure and correcting for every SNP rather than calculating an 'effective number of tests'). Similarly, a set of phenotype-genotype associations is presented, with the appropriate estimate of the FDR. More details can be found in Supplementary material, including Supplementary Figure S2 available online, a visual representation of the correction procedure.

Evidence that the activation of 455 voxels had a genetic component was found, with an associated FDR of 0.5 . This is a highly liberal threshold, but as this first part of the study was intended to contribute to the initial discovery of potential SNPs and significance will be assessed in the replication experiments, a higher proportion of false positives was tolerated to avoid discarding interesting results.

Only clusters of at least 10 voxels were selected. This is a smaller threshold than that used for preselection of the 20,000 
voxels (which insisted clusters had at least 50 voxels), as here we simply wished to discard spurious findings. There were 10 such clusters, totaling 226 voxels, located in the cuneus and occipital gyrus, both known to be involved in visual processing, and bilateral fusiform gyrus, which is implicated as central to face processing specifically. ${ }^{3}$ These clusters were associated with 37 SNPs located in 20 genomic regions $<40 \mathrm{~kb}$. These 20 genetic regions and the associated brain regions are listed in Supplementary Table S1 available online. Supplementary Figures S3-S5 available online show Manhattan and $Q-Q$ plots for three voxel phenotypes, those most significantly associated with SNPs rs12485367, rs16992973 and rs2208796, these are SNPs we will develop further. There is little evidence that any of these SNPs are not in Hardy Weinberg Equilibrium (for rs12485367, $P=1.00$; rs16992973, $P=0.14$; and rs2208796, $P=0.13$ ). We see little evidence of genomic inflation for these phenotypes, for example, $\lambda=0.997$ for the phenotype associated with rs12485367. Supplementary Figures S6-S8 available online shows in more detail the $P$-values for these phenotypes in the genetic regions where they show most significant associations. In Supplementary Figure S6 available online, we see that the association relates to a single signal, as no other SNPs in high linkage disequilibrium with rs 12485367 were available in this dataset. This increases the chance that the association could be a false positive induced by genotyping errors; to rule this out, we have explored the population genetics of this SNP in comparison with the North American sample and the CEU sample from the 1000 genomes pilot project ${ }^{23}$ for evidence of biased ascertainment. This information is contained in the Supplementary Material. We also note that in Supplementary Figure S6 available online, we see that a number of SNPs closest to rs12485367 show $P$-values between $10^{-2}$ and $10^{-4}$ despite being in only weak linkage disequilibrium with rs12485367, some evidence that they may be weakly tagging the causal variant.

We plot genotype against peak phenotype for these three SNPs in Supplementary Figure S9 available online. All three SNPs seem to show a consistent trend across genotype; there is little evidence these associations are induced by a few extreme outliers. Finally, Supplementary Figure S10 available online shows Manhattan and Q-Q plots for the peak voxel associated with each of the 20 genetic regions listed in
Supplementary Table S1 online. Phenotypes from certain brain regions, in particular the cuneus, show more evidence of genomic inflation. This could be because these regions show greater variation in brain activation, possibly breaking the normality assumptions of the model.

Validation of results in positive faces experiment. The 37 most significant SNPs from the negative faces scan were tested in the positive faces scan. This is not an independent replication in a new population, as the experiments were performed on mostly the same people during the same scanning occasion performing a similar task and thus the $P$-values reported are biased towards significance. However, recruitment into the study continued after data for the negative faces scan were collated and analyzed and so genotype data on 57 new individuals were available for the positive faces scan. Two SNPs were discarded as their minor allele frequency was $<0.05$ in this new population, leaving 35 SNPs. Again using an additive model of genetic effect, associations between SNPs in seven genetic regions and neural activation were identified in 26 clusters of $>10$ voxels, located in the cuneus, lingual and superior occipital gyri as well as in the bilateral fusiform gyrus (the associated voxel-wise FDR was 0.05 , the associations with regions of the fusiform gyrus, a structure particularly involved in face processing, are listed in Table 1, all associations discovered are listed in Supplementary Table S2 online).

The neural regions associated with the seven genetic regions across both the negative and positive faces data are all known to be involved in visual processing. Some of these regions, such as the primary occipital gyrus, are involved with general visual processing. Others overlap with the so-called occipital face area which, together with the fusiform gyrus, is considered necessary for normal face processing. ${ }^{24}$ For the fusiform gyrus, the right hemisphere is known to be preferentially involved in face processing. ${ }^{3}$ The results in the negative faces scan are consistent with this, a greater number of associations were found with fusiform gyrus voxels in the right hemisphere than the left hemisphere (36 voxels in the right fusiform gyrus were associated with genetic variants, as opposed to 19 in the left fusiform gyrus). The pattern of activation in the Norwegian sample is particularly striking in the case of rs12485367; significant associations with the

Table 1 SNPs associated with the fusiform gyrus for the positive faces task

\begin{tabular}{llcccc}
\hline SNP & Gene annotation & $\begin{array}{c}\text { Location } \\
\text { (minor allele frequency) }\end{array}$ & Hemisphere & Peak coordinate & Cluster size \\
& & $20: 19048148(0.19)$ & Left & $-22-62-2$ \\
peak significance
\end{tabular}

Abbreviation: SNP, single nucleotide polymorphism.

Three genetic regions from the negative faces task were also associated with activation with the fusiform gyrus in the positive faces task, located in seven clusters of voxels. We present the names and locations of the most significant SNP, the hemisphere within which the association is seen and the most significant nominal $P$-value for association. These associations were further investigated in an independent sample. 
fusiform gyrus, only in the right hemisphere in the negative faces scan (16 voxels), and more pronounced in the right than left hemisphere for the positive faces scan were discovered (linked to 47 voxels in the right hemisphere and 43 in the left). This SNP was not associated with any other brain regions in either experiment. In addition, the peak activation in the right hemisphere to negative faces is in almost exactly the same location as the activation associated with this SNP in the positive faces scan (see Figure 1). In the positive faces experiment, the rs16992973 variant was also associated with bilateral fusiform area activation (though more pronounced in the left hemisphere); the remaining genetic region was associated only with activation in the left fusiform gyrus.

Our initial analysis ignored disease status, as analyses of pilot data found no trend effects on activation. However, as others have found associations between regional brain activation and mental illness, ${ }^{25}$ it was important to investigate whether the present results could be caused by confounding with this factor. We found no significant associations between disease status and the activation of the 20,000 preselected voxels in either experiment. We have also investigated how much controlling for disease status, using indicator variables coding for schizophrenia, bipolar and other psychosis, would affect the results. Supplementary Figure S11 available online shows the $P$-values declared significant in both experiments against the same $P$-values calculated when diagnosis is included in the model (for the negative faces experiment, all associations listed in Supplementary Table S1 available online were reanalyzed, this time regressing on SNP dose and disease status; for the positive faces experiment, all associations listed in Supplementary Table S2 available online were analyzed similarly). We see little change; for both experiments, results were actually slightly improved when diagnosis was included in the model, with a small majority of associations more significant (in the negative faces experiment, 207 associations were more significant including diagnosis, 197 were more significant ignoring it; for the positive faces experiment, these numbers were 627 and 615 , respectively).

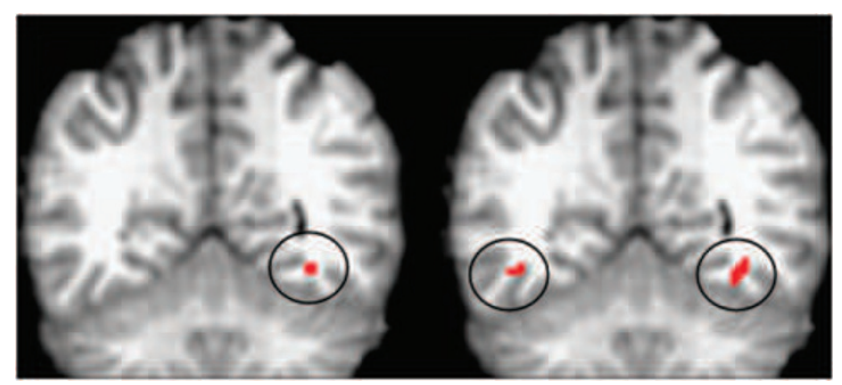

Figure 1 Pattern of activation for rs 12485367 in TMEM212. We present coronal sections ( $Y=-52)$, showing this single nucleotide polymorphism to be associated with activation in fusiform gyrus; unilaterally in the negative faces paradigm displayed on the left, and bilaterally in the positive faces paradigm (panel to right). Activated voxels are circled and colored red. The nominal threshold for significance is $2.1 \times 10^{-8}$ for the negative faces paradigm and $3.2 \times 10^{-4}$ for the positive faces paradigm; after adjusting for multiple testing corresponds to a false discovery rate of 0.5 and 0.05 .
We have also repeated the full analysis performed on the positive faces experiment, but again, controlling for diagnosis. Similarly, we found that the results were largely unchanged, indeed slightly improved. Considering diagnosis makes very little difference to the conclusions we draw and would not affect the choice of SNPs to be investigated further in the replication experiment described below. This agrees with previous work, which found preserved function of the fusiform face area (FFA) in schizophrenia. ${ }^{26}$ These results are covered in more detail in the Supplementary material online.

Replicating fusiform gyrus activations in North American sample. Associations across the negative and positive faces datasets were identified. However, in the first negative faces dataset, we expect half of the associations to be false positives, and in the second positive faces dataset, the estimates of significance are difficult to interpret as the SNPs were selected using related data. Therefore, and in line with recent guidelines for replicating genotype-phenotype associations, ${ }^{27}$ a subset of the significant SNPs was investigated in an independent sample of Caucasians from North America. The replication experiments were designed to concentrate on those SNPs most likely to be involved in face processing. The different levels of complexity of the stimuli could cause activations; viewing faces could also inspire higher levels of attention than geometric figures as could the emotional content. However, there is a substantial body of literature pointing to a specialized role for the FFA in face processing ${ }^{3-6}$ and thus we concentrate our focus on SNPs linked to this brain region, in particular in the positive faces paradigm (one SNP of particular interest, rs12485367, was associated with FFA activation in both negative and positive faces paradigms). We use the literature to argue that activations in this region are related to face processing rather than any other cognitive subtractions. For SNPs associated with other regions, a replicated finding would not have this body of work to aid in the interpretation.

The SNPs that were identified in the negative faces dataset and associated with FFA activation in the positive faces dataset were selected (there were five such SNPs, located in three regions). The analyses on activations in the fusiform gyrus used an anatomical region of interest approach, using standard fMRI analyses, which are impractical in the genomewide association study context due to the large number of tests. The NCI-NHGRI Working Group guidelines state that replication experiments and analyses should be as similar as possible, though this is chiefly in order to remove study differences as a reason for failure to replicate.

The replication sample consisted of healthy middle-aged volunteers who completed a similar fMRI task ${ }^{15-17}$ from which the Norwegian paradigms were derived. These participants were genotyped using the Illumina platform. The SNPs upstream of $S L C 24 A 3(20 p 11.23)$, had a proxy on the Illumina platform (rs6081495). Another SNP, rs16992973 near RP4$764022-001$ (20p12.3), had a proxy in rs6038686. All proxies were in complete linkage disequilibrium $\left(R^{2}\right.$ and $D^{\prime}$ equal 1 in the European ancestry population of the HapMap project $\left.{ }^{28}\right)$. The final SNP, rs12485367 in TMEM212 (3q26.31), did not have a proxy on the Illumina platform and was individually genotyped. 
A significant association between rs12485367 (TMEM212) and right fusiform gyrus activation was obtained $(x=20$, $y=-68, z=-14, t=4.77, P=0.004$, FWE-corrected, $k_{E}=92$; see also Figure 2). As in the original experiments, participants carrying the minor $(G)$ allele showed higher fusiform gyrus activation relative to $C$ homozygotes. Significant association between rs6038686 (RP4-764022-001) and right fusiform gyrus activation $(x=26, y=-52, z=-14 ; T=4.07, P=0.043$, FWE-corrected, $k_{E}=80$; see Figure 3) was also found; such that participants carrying the minor $(G)$ allele showed relatively heightened activation. Again, this matches the result from the Norwegian sample. As in the rs12485367 analyses, the right fusiform gyrus activation remained significant after FWE correction over the search volume of an anatomical fusiform gyrus region of interest. Finally, rs6081495 did not show a significant association with fusiform gyrus activation.

\section{Discussion}

The present findings represent a demonstration of an association between common gene variation and neural function associated with face processing. Validation of some of the initial results using a parallel experiment and replication of the most promising candidates in an independent population was used. It is also important to note that the original associations with fusiform gyrus activation in the Norwegian discovery sample were identified when searching across all neural regions sensitive to the task and not through the narrow window of an anatomical region of interest approach.

There is little knowledge about the function of the genes that were identified, but there are some indications that TMEM212 is expressed in the brain. Affymetrix provides a test of whether a transcript is present or absent within a sample, ${ }^{29}$ and TMEM212 is present in five out of seven samples of the whole brain tissue collated in the Gene Enrichment Profiler ${ }^{30}$ (http:// xavierlab2.mgh.harvard.edu/EnrichmentProfiler/index.html), and in one out of two samples from the occipital lobe.

Interestingly, both genes replicated in the North American sample lie within regions for which associations have been proposed to disorders involving impairment of social functioning. ${ }^{31-35}$ But, as no association to diagnosis was found, and the replication sample excludes any individuals with a current DSM-IV (Diagnostic and Statistical Manual for Mental Disorders) Axis I disorder, we believe these genes to be involved in regulating fundamental brain functions related to face processing unrelated to psychiatric disorders. Further, as these variants were identified and validated using faces expressing negative and positive emotions, these pathways may be broadly important for face processing independent of emotional expression.

The current findings are in line with recent evidence of high heritability of the ability to process face information, ${ }^{12,13}$ which suggests a strong biological control of this important human function. Previous studies indicate that the ability for face
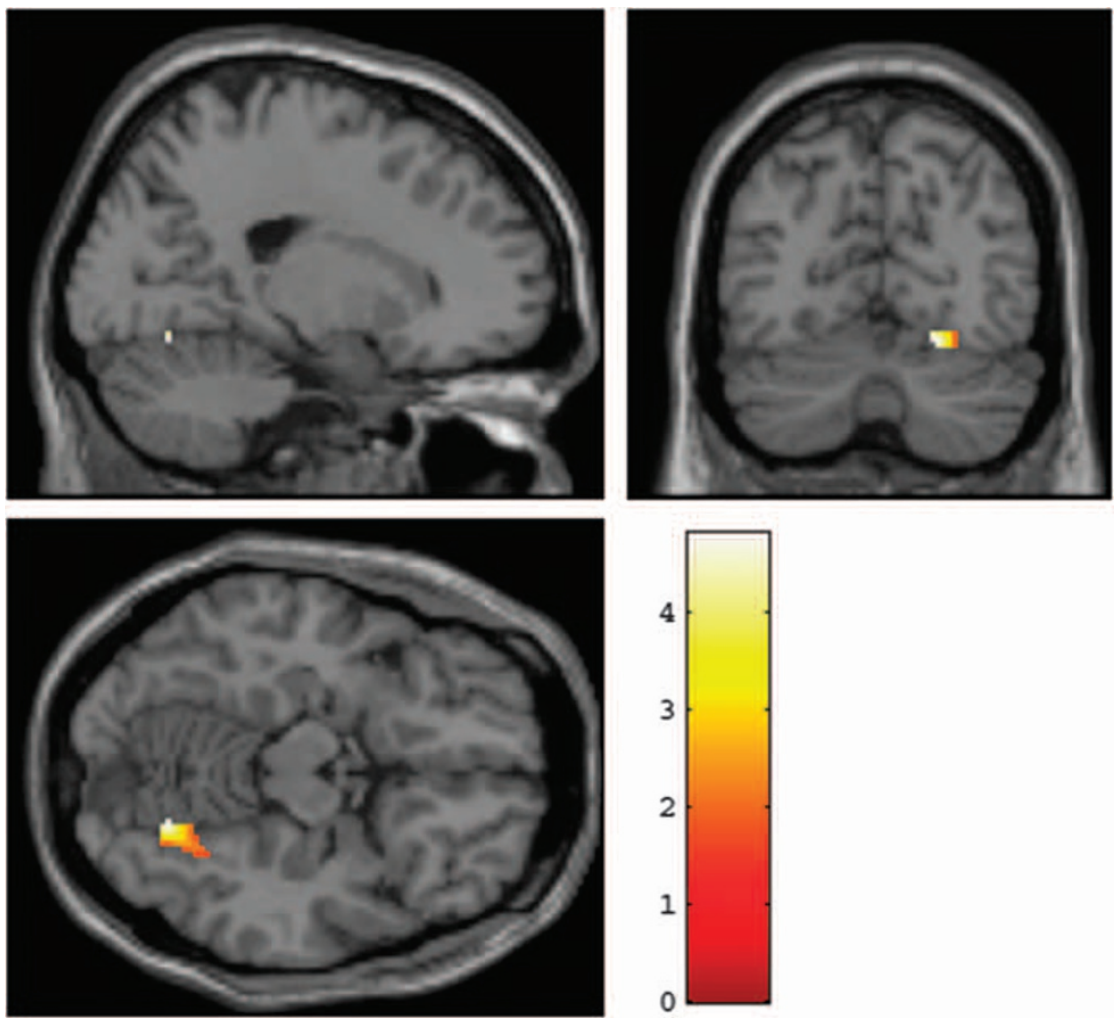

Figure 2 Replication of an association between TMEM212 and fusiform gyrus activation in a North American Caucasian sample. Significant association between rs12485367 and right fusiform gyrus activation is presented in coronal, sagittal and axial overlays on a high-resolution single-subject T1-weighted structural image. Participants carrying the minor $(G)$ allele exhibited relatively heightened activation in comparison to those homozygous for the $C$ allele. Activation parameters: cluster location in MNI (Montreal Neurological Institute) space: $x=20, y=-68, z=-14, T=4.77, P=0.004$, FWE (family-wise error)-corrected, $k_{E}=92$. 

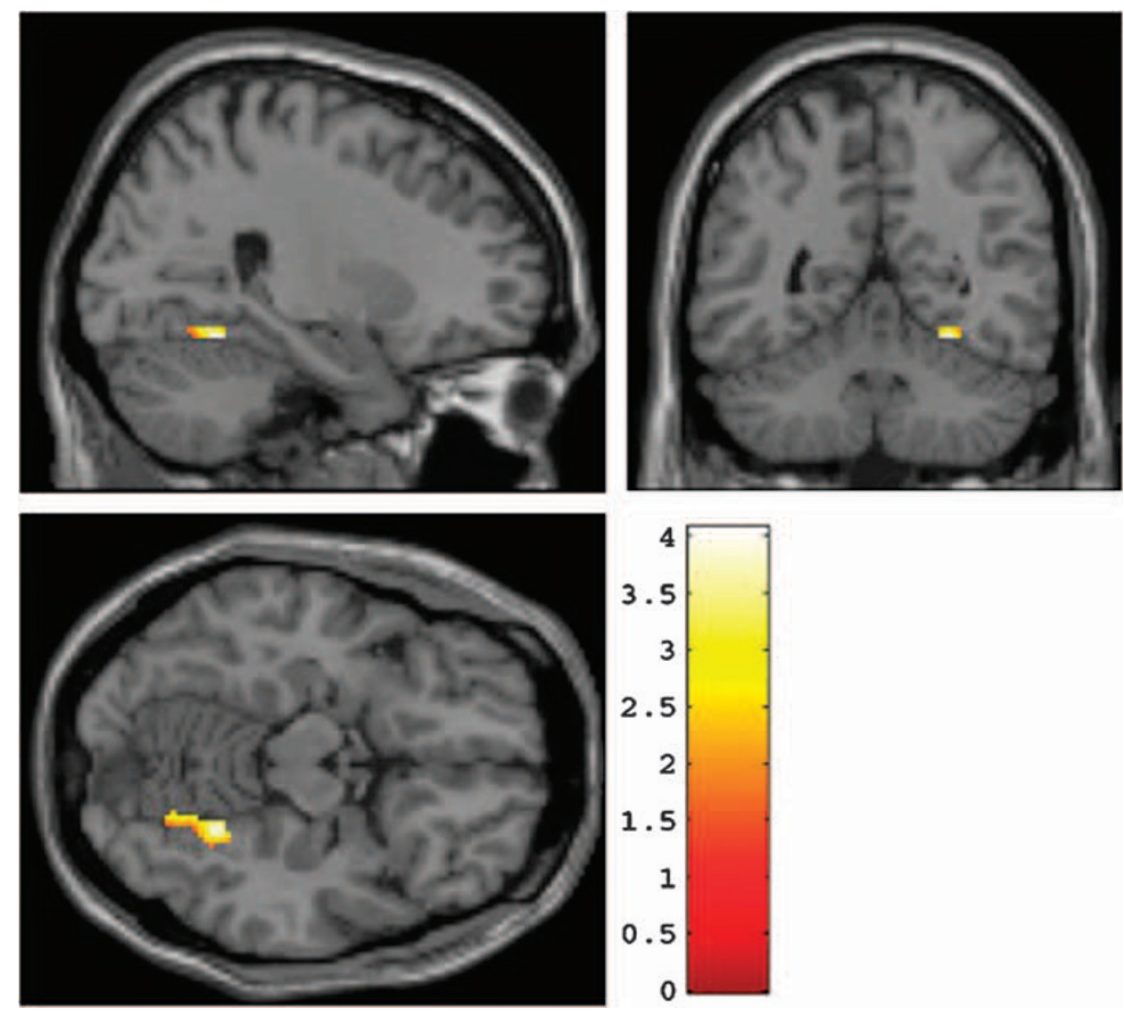

Figure 3 Replication of an association between RP4-764022-001 and fusiform face area response in a North American Caucasian sample. Significant association between rs6038686 and right posterior fusiform response is presented in coronal, sagittal and axial overlays on a high-resolution single-subject T1-weighted structural image. Participants carrying the minor $(G)$ allele exhibited relatively heightened activation in comparison to those homozygous for the $A$ allele. Activation parameters: cluster location in MNI (Montreal Neurological Institute) space: $x=26, y=-52, z=-14 ; T=4.07, P=0.043, k_{E}=80$.

recognition has little overlap with other cognitive abilities, ${ }^{12,13}$ which suggests that the face processing ability is specific. This is supported by the present findings of specific activation patterns related to face processing associated with distinct gene variants, indicating a specific genetic basis. Particularly TMEM212 seems to be specifically involved in the innate architecture of face processing, as it was associated with activation in the FFA only.

This supports the position that distinct cognitive and neuronal mental processes may have specific genetic determinants, as has been seen with language disability. ${ }^{36}$ Thus, the genes identified in the current study would be interesting candidate genes to investigate for association with developmental prosopagnosia, ${ }^{37}$ a disorder characterized by impairments in face recognition that are unaccompanied by brain lesions; multiple cases of this disorder have been seen in the same family. ${ }^{38,39}$ Investigations into pedigrees has suggested that forms of congenital prosopagnosia can have a dominant, autosomal mode of inheritance. ${ }^{40}$ Although we have looked for common variation, these genes could be investigated to find rare variants causing pathological results in such families. However, the development of such distinct mental processes probably depends on geneenvironment interactions.

To conclude, specific genes that may be important for the modulation of basic neurobiological processes, which underlie human social interactions, have been identified. In addition, we have demonstrated how the synthesis of fMRI and genome-wide data can be used to discover and replicate links between common genomic variation and behaviorally relevant brain function.

\section{Conflict of interest}

The authors declare no conflict of interest.

Acknowledgements. We thank the study participants and the members of the TOP study group involved in data collection. This work was supported by Oslo University Hospital, University of Oslo, South-Eastern Norway Health Authority (grant \# 2004-123), and the Research Council of Norway (\#167153/V50, \#163070/ V50). Eli Lilly supported parts of the genotyping costs. We would like to thank Eivind Bakken and Christine Lycke Brandt for their work in processing the data and providing background context, Statistics for Innovation for fruitful discussions and Anders M Dale for his very useful advice and guidance.

1. Farah MJ, Wilson KD, Drain M, Tanaka JN. What is 'special' about face perception? Psychol Rev 1998; 105: 482-498.

2. McKone E, Kanwisher N, Duchaine BC. Can generic expertise explain special processing for faces? Trends Cogn Sci 2007; 11: 8-15.

3. Kanwisher N, Yovel G. The fusiform face area: a cortical region specialized for the perception of faces. Philos Trans R Soc Lond B Biol Sci 2006; 361: 2109-2128.

4. Park J, Newman LI, Polk TA. Face processing: the interplay of nature and nurture. Neuroscientist 2009; 15: 445-449.

5. Ishai A. Let's face it: it's a cortical network. Neuroimage 2008; 40: 415-419.

6. Haxby JV, Hoffman EA, Gobbini MI. The distributed human neural system for face perception. Trends Cogn Sci 2000; 4: 223-233.

7. Tsao DY, Livingstone MS. Mechanisms of face perception. Annu Rev Neurosci 2008; 31: 411-437. 
8. Tsao DY, Moeller S, Freiwald WA. Comparing face patch systems in macaques and humans. Proc Natl Acad Sci U S A 2008; 105: 19514-19519.

9. Farroni T, Mansfield EM, Lai C, Johnson MH. Infants perceiving and acting on the eyes: tests of an evolutionary hypothesis. J Exp Child Psychol 2003; 85: 199-212.

10. Johnson MH, Morton J. Biology and Cognitive Development: The Case of Face Recognition. Blackwell, 1991.

11. Mondloch CJ, Lewis TL, Budreau DR, Maurer D, Dannemiller JL, Stephens BR et al. Face perception during early infancy. Psychol Sci 1999; 10: 419

12. Zhu Q, Song Y, Hu S, Li X, Tian M, Zhen Z et al. Heritability of the specific cognitive ability of face perception. Curr Biol 2010; 20: 137-142.

13. Wilmer JB, Germine L, Chabris CF, Chatterjee G, Williams M, Loken E et al. Human face recognition ability is specific and highly heritable. Proc Natl Acad Sci U S A 2010; 107: 5238-5241.

14. Athanasiu L, Mattingsdal M, Kähler AK, Brown A, Gustafsson O, Agartz I et al. Gene variants associated with schizophrenia in a Norwegian genome-wide study are replicated in a large European cohort. J Psychiatr Res 2010; 44: 748-753.

15. Fakra E, Hyde LW, Gorka A, Fisher PM, Muñoz KE, Kimak M et al. Effects of HTR1A C(-1019)G on amygdala reactivity and trait anxiety. Arch Gen Psychiatry 2009; 66: 33-40.

16. Hariri AR, Gorka A, Hyde LW, Kimak M, Halder I, Ducci F et al. Divergent effects of genetic variation in endocannabinoid signaling on human threat- and reward-related brain function. Biol Psychiatry 2009; 66: 9-16.

17. Manuck SB, Marsland AL, Flory JD, Gorka A, Ferrell RE, Hariri AR. Salivary testosterone and a trinucleotide (CAG) length polymorphism in the androgen receptor gene predict amygdala reactivity in men. Psychoneuroendocrinology 2010; 35: 94-104.

18. Friston KJ, Ashburner JT, Kiebel S, Nichols TE, Penny WD eds.) Statistical Parametric Mapping: The Analysis of Functional Brain. Academic Press: London, 2007.

19. Dudoit S, Shaffer JP, Boldrick JC. Multiple hypothesis testing in microarray experiments. Stat Sci 2003; 18: 71-103.

20. Benjamini Y, Yekutieli D. The control of the false discovery rate in multiple testing under dependency. Ann Stat 2001; 29: 1165-1188.

21. Hariri AR. The neurobiology of individual differences in complex behavioral traits. Annu Rev Neurosci 2009; 32: 225-247.

22. Stein JL, Hua $X$, Lee $S$, Ho AJ, Leow AD, Toga AW et al. Voxelwise genome-wide association study (vGWAS). Neuroimage 2010; 53: 1160-1174.

23. The 1000 Genomes Project Consortium. A map of human genome variation from population based sequencing. Nature 2010; 467: 1061-1073.

24. Rossion B, Caldara R, Seghier M, Schuller AM, Lazeyras F, Mayer E. A network of occipitotemporal face-sensitive areas besides the right middle fusiform gyrus is necessary for normal face processing. Brain 2003; 126: 2381-2395

25. Morris RW, Weickert CS, Loughland CM. Emotional face processing in schizophrenia. Curr Opin Psychiatry 2009; 22: 140-146.

26. Yoon JH, D'Esposito M, Carter CS. Preserved function of the fusiform face area in schizophrenia as revealed by fMRI. Psychiatry Res 2006; 148: 205-216.

27. NCI-NHGRI Working Group on Replication in Association Studies, Chanock SJ, Manolio T, Boehnke M, Boerwinkle E, Hunter DJ et al. Replicating genotype-phenotype associations Nature 2007; 447: 655-660.

28. International HapMap Consortium. The International HapMap Project. Nature 2003; 426 : 789-796.

29. Affymetrix Microarray Suite User Guide. Affymetrix: Santa Clara, CA, 2001.

30. Benita Y, Cao Z, Giallourakis C, Li C, Gardet A, Xavier RJ. Gene enrichment profiles reveal $\mathrm{T}$ cell development, differentiation and lineage specific transcription factors including ZBTB25 as a novel NF-AT repressor. Blood 2010; 115: 5376-5384.

31. Alarcón M, Yonan AL, Gilliam TC, Cantor RM, Geschwind DH. Quantitative genome scan and ordered-subsets analysis of autism endophenotypes support language QTLs. Mol Psychiatry 2005; 10: 747-757.

32. Allen-Brady K, Miller J, Matsunami N, Stevens J, Block H, Farley M et al. A high-density SNP genomewide linkage scan in a large autism extended pedigree. Mol Psychiatry 2009; 14: $590-600$.

33. Arinami T, Ohtsuki T, Ishiguro H, Ujike H, Tanaka $Y$, Morita $Y$ et al. Genomewide highdensity SNP linkage analysis of 236 Japanese families supports the existence of schizophrenia susceptibility loci on chromosomes 1p, 14q, and 20p. Am J Hum Genet 2005; 77: 937-944.

34. Cichon S, Schumacher J, Müller DJ, Hürter M, Windemuth C, Strauch K et al. A genome screen for genes predisposing to bipolar affective disorder detects a new susceptibility locus on 8q. Hum Mol Genet 2001; 10: 2933-2944.

35. Oedegaard KJ, Greenwood TA, Lunde A, Fasmer OB, Akiskal HS, Kelsoe JR et al. A genome-wide linkage study of bipolar disorder and co-morbid migraine: replication of migraine linkage on chromosome 4q24, and suggestion of an overlapping susceptibility region for both disorders on chromosome 20p11. J Affect Disord 2010; 122: 14-26.

36. Fisher SE, Scharff C. FOXP2 as a molecular window into speech and language. Trends Genet 2009; 25: 166-177.

37. Duchaine B, Nakayama K. The Cambridge Face Memory Test: results for neurologically intact individuals and an investigation of its validity using inverted face stimuli and prosopagnosic participants. Neuropsychologia 2006; 44: 576-585

38. Duchaine B, Germine L, Nakayama K. Family resemblance: ten family members with prosopagnosia and within-class object agnosia. Cogn Neuropsychol 2007; 24: $419-430$

39. Lee Y, Duchaine B, Wilson HR, Nakayama K. Three cases of developmental prosopagnosia from one family: detailed neuropsychological and psychophysical investigation of face processing. Cortex 2010; 46: 949-964

40. Grüter T, Grüter M, Carbon CC. Neural and genetic foundations of face recognition and prosopagnosia. J Neuropsychol 2008; 2(Part 1): 79-97.

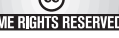

Translational Psychiatry is an open-access journal published by Nature Publishing Group. This work is licensed under the Creative Commons Attribution-Noncommercial-Share Alike 3.0 Unported License. To view a copy of this license, visit http:// creativecommons.org/licenses/by-nc-sa/3.0/

\section{Supplementary Information accompanies the paper on the Translational Psychiatry website (http://www.nature.com/tp)}

